\title{
Electrophysiological Correlates of Emotional See-Saw Effect on a Go/No-Go Task
}

\author{
Remigiusz Szczepanowski ${ }^{1}$ Michal Folwarczny ${ }^{2} \cdot$ Magdalena Król $^{2}$. \\ Dariusz Doliński ${ }^{2} \cdot$ Joanna Budzisz $^{3}$
}

Published online: 30 June 2017

(C) The Author(s) 2017. This article is an open access publication

\begin{abstract}
Social influence studies show that manipulation of rapid affective changes, the so-called emotional see-saw, works effectively as it triggers a mindlessness state through cognitive deficits. This study aimed at investigating the neuronal mechanisms underlying mindlessness behavior evoked by the fear-then-relief technique. We hypothesized that rapid affective changes due to the fear-then-relief procedure impair top-down cognitive operations (monitoring) in the brain. To examine such a hypothesis, we conducted a go/no-go task along with ERP (event-related potential) measures where participants were required to inhibit their motor responses, and then we applied an emotional see-saw to disrupt participants' expectancies regarding their performance. In the next stage, we inspected monitoring operations by analyzing no-go responses and their electrophysiological counterparts in the brain, such as No-Go N2 amplitudes. The electrophysiological measures revealed stronger monitoring impairments in the brain for participants exposed to the emotional see-saw. Contrary to expectations, participants from the emotional see-saw group showed an increase in inhibition as opposed to the group manipulated only with anxiety. One may therefore conclude that there is no clear empirical evidence
\end{abstract}

Remigiusz Szczepanowski

rszczepanowski@uz.zgora.pl

1 Institute of Psychology, The Faculty of Education, Psychology and Sociology, University of Zielona Góra, al. Wojska Polskiego 69, 65 762 Zielona Góra, Poland

2 Faculty of Psychology in Wrocław, SWPS University of Social Sciences and Humanities, Warsaw, Poland

3 Institute of Electrical Power Engineering, Wroclaw University of Science and Technology, Wrocław, Poland concerning distinct behavioral-electrophysiological patterns under an emotional see-saw state.

Keywords Emotional see-saw · Inhibition · Monitoring · Go/no-go task $\cdot$ ERP

\section{Introduction}

There is general agreement among psychologists that, at least from time to time, people behave in a mindless and automatic manner. According to Langer (e.g. Langer 1992; Langer et al. 1978; Pagnini et al. 2016), this usually occurs in routine and recurrent situations. The results of several studies on social influence suggest, however, that a state of mindlessness may also be observed in situations of a sudden and unexpected withdrawal of affective stimulation sources (Dolinski 2001). In a series of studies on social influence it has been demonstrated that under such conditions participants tend to fulfil non-sensical requests and do things against their will (e.g. Nawrat and Dolinski 2007; Dolinski and Szczucka 2012, 2013). More importantly, in one experiment (see: Nawrat and Dolinski 2007) inspired by the influential classic field study on mindlessness by Langer et al. (1978), clear mindless behavior was observed among participants who were jaywalkers. In the control condition, this fact was just recorded, but in the experimental condition participants heard a police whistle blast as they crossed the street. The sound was produced by the experimenter. The participants realized that it was a false alarm and continued crossing the street. Then, each participant was approached by a confederate asking for a donation, either formulating the request without any justification, the request with a real justification, or with a placebic justification. In fact, this study demonstrated that people in emotionally neutral conditions behaved in a thoughtful 
manner - they hardly ever gave money when the request was not accompanied by any justification or when the justification was placebic. However, the fear-then-relief condition was enough for any justification at all to increase the compliance rate. Importantly, in this condition participants hardly ever asked any questions about the aim of the charity campaign and its organizer (Dolinski and Nawrat 1998). In another set of laboratory studies, participants who were subjected to a fear-then-relief scenario took longer to find a particular face in a crowd and solved fewer mathematical tasks than did participants in either the fear-only group or emotionally neutral group (Dolinski et al. 2002).

These results raise the important question of why people comply and behave mindlessly in a state of emotional see-saw - i.e., when a person experiences a state of fear that is then rapidly withdrawn. According to an account by Dolinski and Nawrat (1998) of their findings, it appears that fear invokes a particular program of action (alters posture and body movement, focuses a person's attention on the source of this emotion, etc.). Such reactions are usually adequate for threatening situations. However, this type of reaction ceases to be operative when the circumstances are suddenly reversed, because the source that justifies the fear is in fact withdrawn. In this particular situation, all cognitive efforts are aimed at blocking all current actions induced by the ceased stimulation, in this fashion limiting rationality in decision-making processes (Dolinski 2001, 2007).

Here we challenge this theoretical view showing that rapid affective changes impair top-down operations (monitoring functions), leading in consequence to mindlessness. According to cognitive researchers (Carruthers 2009), topdown operations guide behavior with respect to targets in different situations. In particular, it has been demonstrated that monitoring is a sort of cognition about cognition that constantly probes cognitive processes and their usability to current goals (Carruthers 2009). In other words, monitoring is supposed to verify whether or not current actions are relevant with respect to achieving certain goals of the subject, for instance our expectations. Fernandez-Duque et al. (2000) suggest that monitoring is essential for choosing coherent and successful behavior. If monitoring functions are invalid, then subjects might fare much worse in any task than individuals who successfully monitor the whole set of stimuli (Fernandez-Duque et al. 2000). Thus, it is likely that various situations of compliant behavior induced by sudden withdrawals of affect may be explained by monitoring disruption.

More importantly, it seems that the proper functionality of monitoring strictly affects inhibition. For instance, FernandezDuque et al. (2000) have argued that proper inhibition is crucial as the essential pursuit of metacognitive tasks requires emotions. Thus, it is likely that if monitoring is invalid or impaired at some point, compliant individuals cannot inhibit properly predominant responses when engaged in emotionally-driven tasks. In fact, we can frequently observe such performance in a typical go/no-go task. Under such experimental conditions, in no-go trials the participant is required to withhold response to certain stimuli. For example, in a go/no-go study by Menon et al. (2001) participants were requested to press a button whenever they saw letters other than " $X$ ". In no-go trials, participants were asked to refrain from responding when the letter " $\mathrm{X}$ " appeared. Clearly this experimental procedure enables researchers to measure inhibition, because it requires suppression of an irrelevant proponent stimulus. In addition, following such irrelevant cues leads to mistakes, as indicated elsewhere (see for instance: Fernandez-Duque et al. 2000, which is also diagnostic for inhibition ability.

It is important to note that a vast body of evidence from neurobiological research also shows that the go/no-go task is particularly useful for indicating inhibition in the brain. For instance, several studies have indicated that the anterior cingulate region of the brain is often associated with inhibition when a go/no-go task is performed (Fernandez-Duque et al. 2000). Moreover, some researchers also suggest that inhibitory responses in a go/no-go task are related to the activation of the left premotor areas (Kiefer et al. 1998). Furthermore, Rubia et al. (2001) have found that inhibition tasks are also relevant to the activation of the mesial, medial, and inferior frontal and parietal cortices. Importantly, there are several brain studies (see for instance: Donkers and van Boxtel 2004; Nieuwenhuis et al. 2003) showing that an ERP (eventrelated potential) component such as $\mathrm{N} 2$ is a reliable measure of inhibition. N2 components are typically detected as a negative shift over the fronto-central scalp locations with peak latencies within a range of 200-300 msec (Jodo and Kayama 1992; Nieuwenhuis et al. 2003) or 250-300 msec (Nieuwenhuis et al. 2003). In addition, electrophysiological research based on the go/no-go N2 has identified that there is a strong enhancement for this ERP response in no-go trials as opposed to go trials (Nieuwenhuis et al. 2003). Moreover, when go and no-go trials are presented with equal frequencies, the N2 measures in the brain are still enhanced on the no-go stimuli (e.g. Eimer 1993). Several researchers have also reported that top-down operations of inhibition are responsible for the formation of an increased amplitude of N2 in no-go trials (e.g., Eimer 1993; Jodo and Kayama 1992). This, in turn, may suggest that the N2 amplitudes for the no-go condition could potentially reflect a distortion of top-down cognitive functions in the group of participants exposed to rapid withdrawals of the affect.

Our working hypothesis in this study was that 'see-saw' deficits observed in the social influence domain are largely due to distortion of monitoring functions. Thus, we expected that the top-down operation of inhibition on no-go trials and their corresponding brain responses will be affected by the fear-then-relief technique. Particularly, we hypothesized that 
participants exposed to the fear-then-relief sequence, i.e. receiving negative feedback regarding task performance, and a second piece of information invalidating this feedback would experience distortion of top-down operation in inhibiting motor responses as compared to the fear-induced group with no feedback cancellation. In this manner, we hypothesized that at the neuronal level at the fronto-central scalp locations the fearthen-relief group will show diminished no-go N2 amplitudes as opposed to brain activity in the fear-induced group where monitoring functions were left unchanged.

\section{Methods}

\section{Participants}

Participants were 40 healthy right-handed undergraduates of the SWPS University of Social Sciences and Humanities, Faculty of Psychology in Wroclaw, with normal or corrected-to-normal vision (7 males and 33 females), taking part in the study for course credits. Their mean age was 25.6 $(\mathrm{SD}=6.8)$ and ranged from 19 to 43 . They were recruited using an advertisement within the University's internal IT system saying that they could participate in a study of facial perception. Participants were randomly assigned to both experimental groups, with participants assigned an odd number taking part in the fear-induced condition, while participants with an even number took part in the fear-then relief condition. The study exclusion criteria were no current or previous history of neurological or psychiatric conditions. Datasets from four participants were removed due to the poor quality of their EEG recordings. All volunteers gave their written consent to take part in the study and were fully debriefed after its completion. The study protocol was approved by the local SWPS University Ethics Committee in accordance with the Helsinki Declaration.

\section{Stimuli}

The present study employed two sets of facial stimuli which were elaborated by researchers from the Karolinska Institute (Karolinska Directed Emotional Faces: Goeleven et al. 2008) and a second set developed by researchers from the Laboratory of Cognition and Emotion (Indiana University, USA). The whole set of faces contained 60 female and 60 male neutral facial expressions that were displayed in a random order. Each face was presented only once per session. Participants' heads were fixed with a chin rest. Stimuli were presented on a 22-in. Iiyama ProLite E2208HDSV display with $1920 \times 1080$ resolution. Participants were seated in a dim room, $60 \mathrm{~cm}$ from the screen presenting the stimuli with their right hand resting on the space key, which they were requested to press when the relevant stimulus appeared. Our experiment was programmed in Matlab, using the Psychophysics Toolbox extensions (Brainard 1997; Pelli 1997; Kleiner et al. 2007).

\section{Go/No-go Task}

The go/no-go task involving response conflicts was used in the present study (see Fig. 1). In each trial, after presenting a fixation cross for $100 \mathrm{msec}$, the participants were cued by a male or female neutral face target for $100 \mathrm{msec}$, prompting them to make a rapid response (the go condition) or withhold a response (the no-go condition). In the go condition, participants were asked to produce a rapid response with the dominant hand using the space bar on the numerical keyboard for a fixed time. The time interval for responses was randomized within a range of 1300-1700 msec with an interval of $100 \mathrm{msec}$ to avoid habituation. In such conditions, it was expected that greater effort was required to produce no-go responses. The go and no-go trials were presented with equal frequencies (50/50), and the order of the stimuli presentations was randomized for each participant. The participants attended two sessions of the go/ no-go task, i.e. pre- and post-test conditions. Each session consisted of four blocks, and each block involved 60 trials (30 male and 30 female faces). In this fashion, a total number of 120 no-go trials was yielded in preand post-test conditions, respectively. At the start of each block, a male or female facial cue was presented prompting participants whether they should withhold (no-go) or generate a rapid (go) response. The preand post-conditions of the go/no-go task lasted about $7 \mathrm{~min}$ and $30 \mathrm{~s}$ each.

\section{EEG Recording}

EEG data were acquired via the Quick Amp EEG system (Brain Products GmbH, Munich, Germany), from 64 Ag/ $\mathrm{AgCl}$ electrodes using an ActiCap electrode cap (Brain Products $\mathrm{GmbH}$, Munich, Germany) placed according to the International 10-20 system, relative to the standard reference electrode located at $\mathrm{FC}_{\mathrm{z}}$ and the ground reference located at $\mathrm{AF}_{\mathrm{z}}$. Electrooculograms were recorded using a pair of bipolar electrodes. In particular, VEOG electrodes were placed above and below the right eye, and HEOG electrodes were placed at the outer canthi of each eye. Impedances were kept below $5 \mathrm{k} \Omega$. Data were digitized at a sampling rate of $250 \mathrm{~Hz}$.

All data processing was performed using the Brain Electrical Source Analysis (BESA 2000, version 6.0; BESA GmbH, Gräfelfing, Germany). The data were filtered between $0.05 \mathrm{~Hz}$ and $30 \mathrm{~Hz}$ and re-referenced to the average reference. Ocular artifacts were corrected using the standard BESA procedure. The data were segmented into epochs of $2300 \mathrm{~ms}$ 
Fig. 1 Diagram of sequence in the go/no-go task

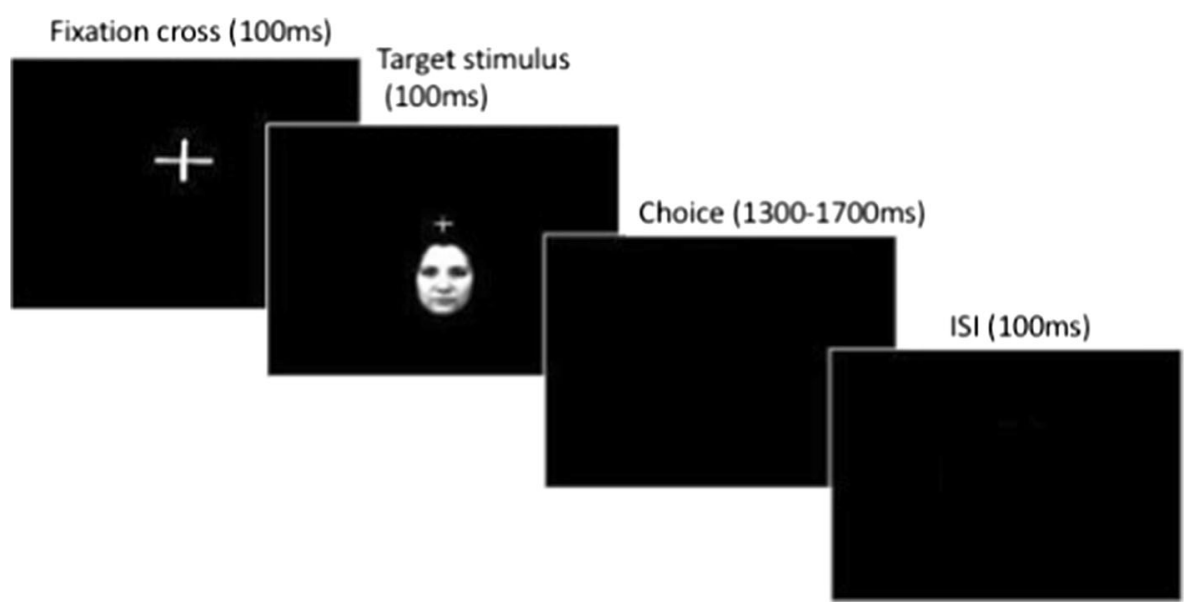

(800-ms pre-stimulus to $1500 \mathrm{~ms}$ post-stimulus) and corrected to a 200-ms pre-stimulus baseline. Epochs containing artifacts with amplitudes exceeding $120 \mu \mathrm{V}$ and with a gradient of $75 \mu \mathrm{V}$ were rejected from the signal. Data from four participants were rejected entirely due to excessive noise and poor signal quality.

\section{Procedures}

At the beginning of the study, volunteers were randomly assigned to one of two experimental conditions: the fearinduced (FI) group and fear-then-relief (FR) group. Each participant was then prepared for the EEG data recordings, and then right after underwent a short training session with the experimenter to familiarize themselves with the go/no-go task. Each participant was required to make go responses as quickly as possible. The participants attended two experimental sessions of the go/no-go task, where the first session served as a baseline while the second one was intended to measure monitoring distortion in the brain as a result of the emotional seesaw procedure administration. Thus, after completing the baseline go/no-go session, a message appeared on the computer screen which informed participants about their performance in the go-no/go task. Regardless of participants' performance, the message indicated that performance was lower than expected. Since the go/no-go task was relatively easy to perform, it was assumed that receiving an opposite-thenexpected feedback regarding task performance would induce negative feelings in participants. Then, in the fear-then-relief experimental condition the experimenter explained to participants that the message was a computer program error and asked participants to complete the second session of the go/ no-go task. In the fear-induced condition, participants were only required to complete the second go/no-go session. After the whole study was completed a debriefing procedure began via an e-mail with a full explanation of the experimental procedure. The experimental manipulation along with presentation of feedback on the screen lasted around $10 \mathrm{~s}$.

\section{Statistical Analysis}

At the behavioral level it was expected that participants exposed to the fear-then-relief sequence would exhibit a decline in inhibition ability as compared to the fear-induced group with no feedback cancellation administered. We used the number of correct no-go responses as the dependent variable. Separate repeated measures analyses of variance (ANOVA) were performed on the behavioral no-go responses and their ERP counterparts. The behavioral data were submitted into a repeated measures analyses of variance (ANOVA) comparing no-go responses between two groups (FI vs. FR) across two within-subject factors such as the learning factor, and the face gender factor (male vs. female face).

At the EEG level, we established the signal of NoGo N2 as the dependent variable as recommended by Nieuwenhuis et al. (2003), and the N2 indicated inhibition activity in the go/nogo task. As for the FR group, we expected a lower amplitude of the EEG signal indicating the decline in ability to inhibit motor responses as compared to the fear-induced group. In terms of the ERP data analysis, time windows for N2 peak extraction were established based on visual inspections of the data yielded at a latency of 200-280 msec. Finally, separate repeated-measures ANOVAs at the locations of $F_{\mathrm{z}}$ and $C_{\mathrm{z}}$ were performed comparing no-go N2 amplitudes between the FI and FR groups, taking into account pre- and postactivity in the brain and the gender of the stimuli.

\section{Results}

\section{Behavioral Responses}

First, we examined the emotional see-saw effects on inhibition ability by inspecting the correct no-go trials. Outcome variables were checked for normality as shown in Table 1 . The parameters of skewness, kurtosis, and the significance level of the Shapiro-Wilk test were calculated (see Table 1). Due to ceiling 
Table 1 Descriptive parameters for correct no-go responses collapsed into the raw and log-transformed data along with the normality tests

\begin{tabular}{|c|c|c|c|c|c|c|c|c|c|}
\hline \multirow{2}{*}{\multicolumn{2}{|c|}{ Dependent Variables(Y) }} & \multicolumn{4}{|l|}{ Pre-test } & \multicolumn{4}{|l|}{ Post-test } \\
\hline & & \multicolumn{2}{|c|}{ Fear-induced } & \multicolumn{2}{|c|}{ Fear-then-Relief } & \multicolumn{2}{|c|}{ Fear-induced } & \multicolumn{2}{|c|}{ Fear-then-Relief } \\
\hline \multicolumn{2}{|l|}{ Data set } & Male & Female & Male & Female & Male & Female & Male & Female \\
\hline \multirow[t]{5}{*}{ Raw data } & Mean & 23.11 & 21.64 & 24.08 & 22.50 & 24.25 & 20.39 & 24.89 & 21.97 \\
\hline & $\mathrm{SD}$ & -.35 & 4.27 & 2.87 & 4.78 & 3.97 & 4.81 & 2.68 & 5.09 \\
\hline & Skewness & -1.43 & -1.10 & -.35 & -1.13 & -2.13 & -.75 & -1.13 & -.91 \\
\hline & Kurtosis & 1.71 & 2.24 & -.20 & 1.35 & 7.16 & .31 & 1.29 & .19 \\
\hline & $\mathrm{W}-\mathrm{S} p$ & $.000 * * *$ & $.021 *$ & .59 & $.009 * *$ & $.000 * * *$ & .095 & $.005 * *$ & $.009 * *$ \\
\hline \multicolumn{10}{|c|}{ Log-transformed } \\
\hline & Mean & .85 & .92 & .80 & .86 & .76 & .98 & .65 & .87 \\
\hline & SD & .20 & .23 & .20 & .27 & .25 & .21 & .24 & .26 \\
\hline & Skewness & .29 & -1.62 & -.73 & -.79 & -.62 & -.33 & -.42 & -.25 \\
\hline & Kurtosis & -.07 & 6.2 & .30 & 1.95 & 2.02 & -.19 & .61 & -.42 \\
\hline & $\mathrm{W}-\mathrm{S} p$ & .226 & $.001 * *$ & .092 & .116 & .066 & .719 & .254 & .588 \\
\hline
\end{tabular}

For $\log$-transformation of variables the formula of $(\log 10(1+\mathrm{K}-\mathrm{DV}))$ was used, where DV is the dependent variable and $\mathrm{K}$ is a constant with each score subtracted (Tabachnick and Fidell, 2007); W-S $p$ indicates the significance level of the Wilk-Shapiro test for normality of the raw and log-transformed data; note that insignificant $p$-values in bold indicate that the corresponding variables are normally distributed; $*-p<0.05 ; * *-p<0.01 ; * * *-p<0.001$

effects of our paradigm the observed data was systematically negatively skewed. Since the dependent variables were nonnormally distributed, we applied a log-transformation formula to the raw data. The outcome variables were log-transformed according to a formula of $\log 10(1+\mathrm{K}-\mathrm{DV})$, where $\mathrm{DV}$ is a dependent variable and $\mathrm{K}$ is a constant (Tabachnick and Fidel, 2007). All observed variables but one benefited from logarithmic transformation work and passed the Wilk-Shapiro normality test. We therefore ran an analysis of variance (ANOVA) that was relatively robust to modest violations of normality assumptions.

In the next step, to compare the go/no-go effects between two groups (i.e. FI and FR groups) a repeated-measures ANOVA was performed, where the two within-subject factors were inhibition ability (pre- vs. post-test condition) and the gender cue (male vs. female face). The analysis showed a significant effect of the group factor, $F(1,70)=6.31, p<.05$, partial eta squared $=.08$, indicating that the emotional see-saw affected inhibition to a greater extent in the FI group $(\mathrm{M}=.879$, $\left.\mathrm{M}_{\text {inversed }}=23.43\right)$ as compared to the FR group $(\mathrm{M}=.799$, $\left.M_{\text {inversed }}=24.7\right)$. Note that the variables were inversely transformed to indicate the original data. The within-subject analysis indicated no learning effect, $F(1,63)=3.53, p=.07$, and no interaction between the group factor and learning factor, $F(1$, $70)=1.39, p>.05$, and the gender factor, $F<1$. Further posthoc analysis with Bonferroni adjustments $(p<0.01)$ indicated a significant difference in inhibition (see Fig. 2) between the fearthen-relief and the fear-induced groups for the post-test condition $(\mathrm{M}=-0.10, \mathrm{SD}=0.04)$ as opposed to the pre-test condition $(\mathrm{M}=-0.06, \mathrm{SD}=0.04)$. In addition, a Bonferroni test $(p<.05)$ showed that for the FR group there was a significant increase in inhibition between the pre- and post-test conditions $(\mathrm{M}=-.06$,
$\mathrm{SD}=.03)$. ANOVA also showed a main effect of the gender nogo stimuli, $F(1,70)=17.83, p<.0001$, partial eta squared $=.20$, while the interaction between the learning and gender variables, $F(1,70)=16.28, p<.001$, partial eta squared $=.19$. Multiple comparisons with the Bonferroni adjustments $(p<.05)$ indicated that there was a stronger inhibition effect in the post-test condition for the female cue as compared to the male cue, $\mathrm{M}=0.22$, $\mathrm{SD}=0.04$; for the pre-test condition this difference was not significant, $\mathrm{M}=0.07, \mathrm{SD}=0.04$. No other second-order interaction with learning and gender factors and the between-subject variable was observed. Contrary to our initial hypothesis, these behavioral results indicated that the fear-induced group could experience stronger distortion in the top-down operation of inhibition as opposed to the fear-then-relief group.

\section{Analysis of No-go N2 Component}

In the next step, we began an analysis of no-go N2 components in order to investigate the electrophysiological components of inhibition. Fig. 3 shows the overall characteristics of N2 waveforms for no-go trials. Visual inspection clearly indicated negativity of the N2 components over the fronto-central scalp locations with a peak between 200 and 280 msec. First, we submitted the ERP data to a repeated-measures ANOVA to compare no-go N2 amplitudes between the two groups (i.e. fear-induced and fear-then-relief) across two within-subject factors such as inhibition ability (pre- vs. post-condition) and the gender factor (male vs. female face no-go stimuli) at the electrode location of $F_{\mathrm{z}}$. The analysis indicated no effect of experimental manipulation on ERP activity, $F<1$. However, the analysis showed that there was an interaction between the group and inhibition 
Fig. 2 Inhibition across FI and FIR groups for the logtransformed data. The y-axis shows the associated correct responses in the no-go condition. Diminished inhibition ability in post-test condition for emotional see-saw group was observed

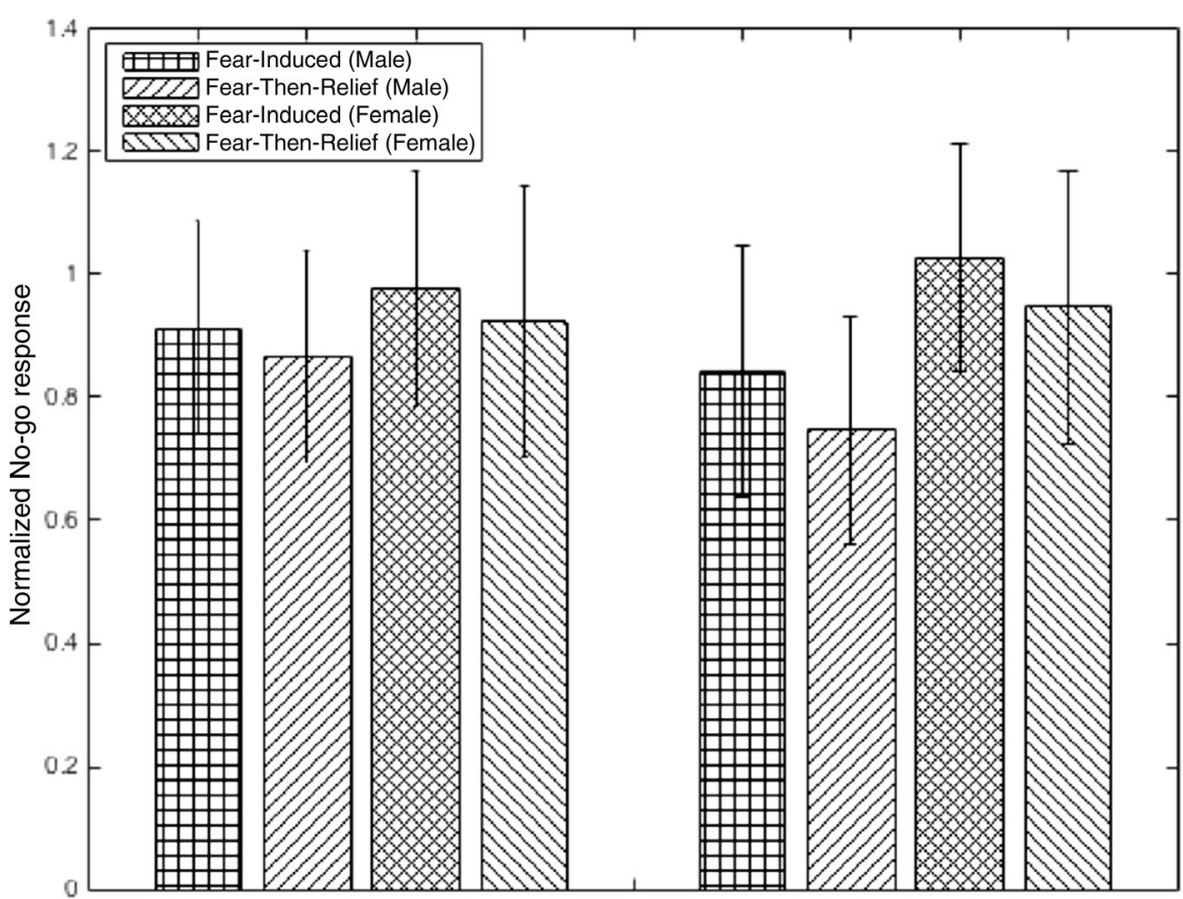

Pre-test condition

Post-test condition activity, $F(1,34)=6.03, p<.05$. Within-subject analysis also showed a main effect for the inhibition activity, $F(1,34)=17.03, p<.001$ where the mean value of the brain activity was lower for the pre-test $(\mathrm{M}=-5.60[\mu \mathrm{V}]$, $\mathrm{S}=0.50[\mu \mathrm{V}])$ as compared to the post-test $(\mathrm{M}=-6.23[\mu \mathrm{V}]$, $\mathrm{S}=0.47[\mu \mathrm{V}])$ conditions. To further investigate the interaction between inhibition activity and the type of group, we performed multiple comparisons using Bonferroni adjustments. Post-hoc analyses $(p<.05)$ showed that for the FI group there was a significant difference between pre- $(\mathrm{M}=-5.41[\mu \mathrm{V}]$, $\mathrm{SD}=0.67[\mu \mathrm{V}])$ and post-test $(\mathrm{M}=-6.43[\mu \mathrm{V}], \mathrm{SD}=0.67$ $[\mu \mathrm{V}])$ conditions as opposed to the FR group where there was no difference between pre- $(\mathrm{M}=-5.78[\mu \mathrm{V}], \mathrm{SD}=0.71[\mu \mathrm{V}])$ and post-test $(\mathrm{M}=-6.04[\mu \mathrm{V}], \mathrm{SD}=0.67[\mu \mathrm{V}])$. No other factor or interaction reached significance. We then performed the repeated-measures ANOVA at the next front-central location, which was the $C_{\mathrm{z}}$ electrode site. The analysis revealed the strong effect of inhibition activity between the pre- and post-test conditions, $F(1,34)=14.36, p<.001$ yielding a mean value of activity for the pre-test of $\mathrm{M}=-3.02[\mu \mathrm{V}](\mathrm{SD}=0.36[\mu \mathrm{V}])$, and $\mathrm{M}=-3.62[\mu \mathrm{V}](\mathrm{SD}=0.35[\mu \mathrm{V}])$. No other factor or interaction reached significance.

\section{Discussion}

The present study investigated a hypothesis with the more detailed assumption that rapid changes of affective state induced by the emotional see-saw technique affects top-down operations in the brain. To test this assumption, we employed a computer cognitive task within the go/no-go paradigm with ERP recordings to measure monitoring distortion while processing information in the brain. We examined ERP components such as N2 responses that have traditionally been associated with the inhibition of motor responses while having participants perform a go/no-go task (e.g. Eimer 1993). Our study based on behavioral no-go measures indicated that participants exposed to an emotional see-saw revealed weaker impairments of inhibition as opposed to cognitive functioning of the group manipulated only with a state of fear (socialevaluative anxiety). In addition, ERP analysis indicated that the brain activity linked with the no-go N2 components underlying the top-down operation of inhibition was diminished for people who experienced the fear-then-relief sequence.

We have hypothesized that participants exposed to the fearthen-relief sequence would demonstrate higher impairment in top-down operations in the brain than participants in the fear condition. This reasoning was based on the assumption that in the fear-then-relief state all cognitive efforts are aimed at blocking actions induced by ceased stimuli, while at the same time they are aimed at increasing cautiousness toward a quite new and totally unexpected external situation. This mental activity would produce cognitive depletion. As a consequence of experiencing this particular state, a person should demonstrate an impairment in a subsequent cognitive task. Contrary to this expectation, however, the analysis of the results of our study revealed that the fear-then-relief participants performed better on the task than did participants in the fear-induced condition. On the other hand, the aim of this study was to test at the electrophysiological level the influence of the emotional 
Fig. 3 Grand-averages of ERPs for FI and FR groups at electrode $C z$ and $F z$. ERP activity for baseline condition depicted with dashed lines; ERP activity for post-test condition marked with dashed lines

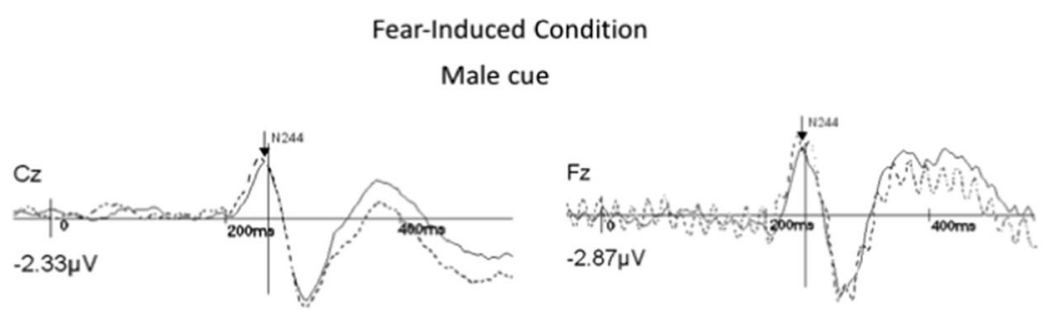

Female cue

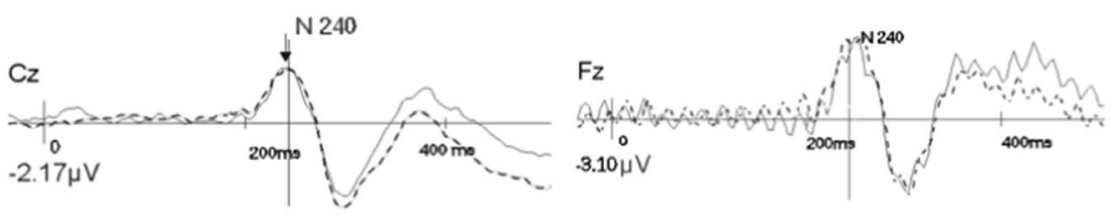

Fear-then-Relief Group

Male cue

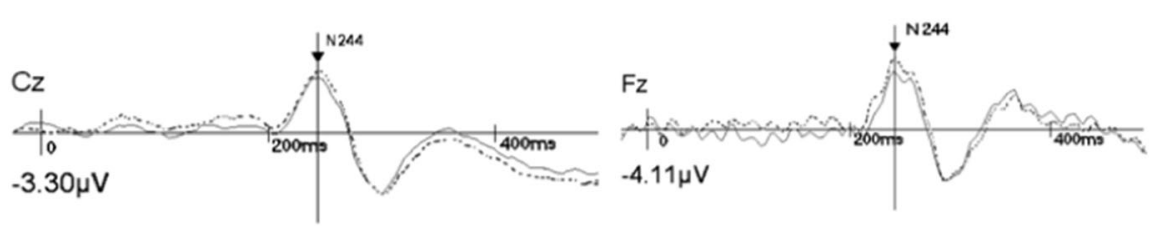

Female cue
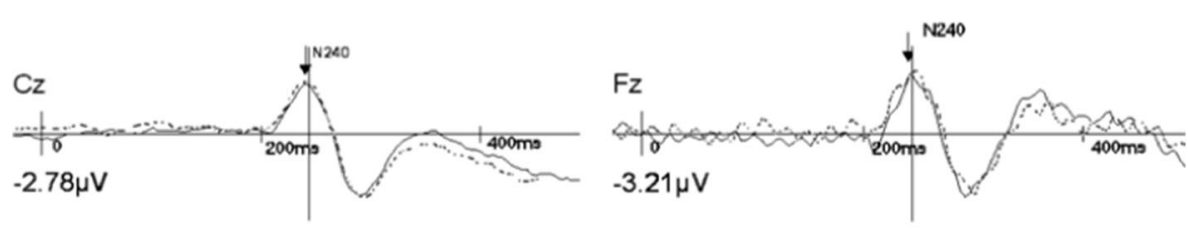

Post-test cond. Pre-test cond.

see-saw technique on top-down operations in the brain. Consequently, diminished neuronal activity in the brain required for the inhibition of motor responses was expected to be present for the fear-then-relief condition as compared to the fear-induced condition. In line with this second part of our hypotheses we found that the cortical activity underlying inhibition for no-go trials was effectively modulated by a sudden withdrawal of emotions at peaks around of 200-280 msec after the stimulus onset. For the fear-the-relief condition we observed a decrease of negativity $\mathrm{N} 2$ at the frontal locations after the administration of an emotional see-saw, indicating the presence of monitoring impairments. How can these results be explained given such a lack of correspondence of behavioral performance?

To understand this pattern of results, it should be taken into account that cognitive engagement does not always lead to cognitive depletion (e.g. Wickens 2002, 2007). In particular, if the initial activity is intensive but at the same time not extremely energy-drying, cognitive warm-up and improvement in functioning rather than cognitive depletion and impairment is observed (e.g., Spiewak 2006, 2013). While in a series of experiments concerning the role of the fear-then-relief phenomenon in social influence processes the manipulation of emotional states was strong or even dramatic (e.g. people were asked if they had just lost their wallet in Dolinska and Dolinski's [2014] study, or were informed they would be given an electric shock while learning in Dolinski and Nawrat's [1998] study), in the experiment presented here this manipulation was rather subtle - participants were informed that their performance in the task (which was not very important for them) was worse than they expected. Since in such situations one should expect an occurrence of the cognitive warm-up effect and an increase in the availability of cognitive resources, it is reasonable that our fear-then-relief participants performed better on the cognitive task than did participants in the fear condition.

Given that electrophysiological measures do not support differences in overt performance in the go/no-go task, there 
is a need to provide another alternative explanation for the observed behavioral-electrophysiological patterns due to emotion see-saw manipulation. For deeper consideration of the precise nature of the presented patterns, it is important to indicate that negative emotion see-saw manipulation (Dolinski and Nawrat 1998) engages processing of external information associated with social-evaluative anxiety that dramatically changes attention to internally processed cues (Clark and Wells 1995). Thus, in the presence of social-evaluative cues, high-anxiety individuals are biased towards processing external information associated with social-evaluative anxiety, which in fact leads them to the faster reactions to a stimulus on their body than to external stimulus as opposed to individuals with low levels of social anxiety who present no bias towards external processing (Mansell et al. 2003). In addition, it is well-established that anxiety impairs goal-directed attentional systems and makes people focus more on threat-related stimuli, which generally leads to impaired performance of a task (Eysenck et al. 2007). In fact, a recent EEG study also reports that higher anxiety is linked with increased negativity of the $\mathrm{N} 2$ component, especially in the conditions of error detection and response monitoring, similar to the conditions in our study (Righi et al. 2009). In particular, the researchers in this study showed that the N2 amplitude increased in individuals with higher anxiety scores, who were more anxious and less confident in order to achieve a better performance in the go/no-go task. Indeed, since our emotion see-saw manipulation involved social-evaluative anxiety, the presented behavioralelectrophysiological activity evoked by the no-go stimulus follows similar patterns. The increasing negativity of the N2 component along with weakened overt performance indicate that the fear-induced group experienced a higher level of anxiety. This, in turn, given the presence of the same cognitive efficiency before the emotion see-saw procedure, could indicate that participants were less anxious and therefore less focused on processing socially-evaluative cues and presented normal inhibition to motor reactions.

It is worth stressing that the research presented in this article is the first attempt to investigate the see-saw phenomenon at the electrophysiological level, but is not fully conclusive. Data obtained in previous research were behavioral in nature, e.g. participants experiencing a relief state fulfilled absurd requests (e.g. Nawrat and Dolinski 2007), did not ask for additional information (Dolinski and Nawrat 1998) or made errors while solving arithmetic equations (Dolinski et al. 2002). Unfortunately, the present electrophysiological research does not provide decisive empirical support for the supposition that a sudden withdrawal of affective stimulation may disable proper monitoring functions. As already suggested, cognitive warm-up and improvement in functioning could possibly obscure expected performance in the fear-the relief group, resulting in more effective inhibition. In fact, the observed lack of correspondence between overt performance and electrophysiological measures confirm findings from previous studies investigating the effects of anxiety on cognition and performance on a go/no-go task (Righi et al. 2009). Our data again shows that the $\mathrm{N} 2$ component can be a reliable index of top-down operations encompassing response inhibition for no-go trials (Eimer 1993; Jodo and Kayama 1992) (Donkers and van Boxtel 2004) when exposed to sociallyevaluative situations. Thus, further investigation needs to be pursued to reveal whether monitoring and inhibition processes play a pivotal role in the domain of social influence.

Acknowledgements This research has been supported by the National Science Center (Poland), and funded under grant decision DEC-2011/03/ B/HS6/01799 to R.S.

\section{Compliance with Ethical Standards}

Funding This study was funded by the National Science Center (grant number DEC-2011/03/B/HS6/01799).

Ethical Approval All procedures performed in studies involving human participants were in accordance with the ethical standards of the institutional and/or national research committee and with the 1964 Helsinki declaration and its later amendments or comparable ethical standards.

Informed Consent Informed consent was obtained from all individual participants included in the study.

Open Access This article is distributed under the terms of the Creative Commons Attribution 4.0 International License (http:// creativecommons.org/licenses/by/4.0/), which permits unrestricted use, distribution, and reproduction in any medium, provided you give appropriate credit to the original author(s) and the source, provide a link to the Creative Commons license, and indicate if changes were made.

\section{References}

Brainard, D. H. (1997). The Psychophysics Toolbox. Spatial Vision, 10, 433-436.

Carruthers, P. (2009). How we know our own minds: The relationship between mindreading and metacognition. Behavioral and Brain Sciences, 32(2), 121-138.

Clark, D. M., \& Wells, A. (1995). A cognitive model of social phobia. In M. Liebowitz, D.A. Hope, E. Schneier, \& R.G. Heimberg (Eds.) Social phobia: diagnosts, assessment and treatment. New York: Guilford Press.

Dolinska, B., \& Dolinski, D. (2014). Fear-then-relief, legitimizing paltry contribution, and charity. European Review of Applied Psychology, 64(1), 29-34.

Dolinski, D. (2001). Emotional see-saw, compliance, and mindlessness. European Psychologist, 6(3), 194-203.

Dolinski, D. (2007). Emotional see-saw. In. A.R. Pratkanis (Ed.) The science of social influence: Advances and future progress. (pp. 137-153). New York and Hove: Psychology Press.

Dolinski, D., \& Nawrat, R. (1998). "Fear-then-relief" procedure for producing compliance: beware when the danger is over. Journal of Experimental Social Psychology, 34(1), 27-50. 
Dolinski, D., \& Szczucka, K. (2012). Fear-Then-Relief-Then Argument. How to Sell Goods Using the EDTR Technique of Social Influence. Social Influence, 7, 251-267.

Dolinski, D., \& Szczucka, K. (2013). Emotional Disrupt-Then-Reframe and compliance. Journal of Applied Social Psychology, 43, 20312041.

Dolinski, D., Ciszek, M., Godlewski, K., \& Zawadzki, M. (2002). Fearthen-relief, mindlessness, and cognitive deficits. European Journal of Social Psychology, 32(4), 435-447.

Donkers, F. C., \& van Boxtel, G. J. (2004). The N2 in go/no-go tasks reflects conflict monitoring not response inhibition. Brain and Cognition, 56(2), 165-176.

Eimer, M. (1993). Effects of attention and stimulus probability on ERPs in a Go/Nogo task. Biological Psychology, 35(2), 123-138.

Eysenck, M. W., Derakshan, N., Santos, R., \& Calvo, M. G. (2007). Anxiety and cognitive performance: attentional control theory. Emotion, 7(2), 336

Fernandez-Duque, D., Baird, J. A., \& Posner, M. I. (2000). Executive attention and metacognitive regulation. Consciousness and Cognition, 9(2), 288-307.

Goeleven, E., De Raedt, R., Leyman, L., \& Verschuere, B. (2008). The Karolinska directed emotional faces: a validation study. Cognition and Emotion, 22(6), 1094-1118.

Jodo, E., \& Kayama, Y. (1992). Relation of a negative ERP component to response inhibition in a $\mathrm{Go} / \mathrm{No}-\mathrm{go}$ task. Electroencephalography and Clinical Neurophysiology, 82(6), 477-482.

Kiefer, M., Marzinzik, F., Weisbrod, M., Scherg, M., \& Spitzer, M. (1998). The time course of brain activations during response inhibition: evidence from event-related potentials in a go/no go task. Neuroreport, 9(4), 765-770.

Kleiner M, Brainard D, Pelli D. (2007). "What's new in Psychtoolbox3?" Perception 36 EVCP. Abstract Suplement.

Langer, E. J. (1992). Matters of mind: Mindfulness/mindlessness in perspective. Consciousness and Cognition: An International Journal, 1, 289-305.

Langer, E. J., Blank, A., \& Chanowitz, B. (1978). The mindlessness of ostensibly thoughtful action: The role of "placebic" information in interpersonal interaction. Journal of Personality and Social Psychology, 36, 635-642.
Mansell, W., Clark, D. M., \& Ehlers, A. (2003). Internal versus external attention in social anxiety: An investigation using a novel paradigm. Behaviour research and therapy, 41(5), 555-572.

Menon, V., Adleman, N. E., White, C. D., Glover, G. H., \& Reiss, A. L. (2001). Error-related brain activation during a Go/NoGo response inhibition task. Human Brain Mapping, 12(3), 131-143.

Nawrat, R., \& Dolinski, D. (2007). "Seesaw of emotions" and compliance: Beyond the fear then-relief rule. The Journal of Social Psychology, 147(5), 556-571.

Nieuwenhuis, S., Yeung, N., Van Den Wildenberg, W., \& Ridderinkhof, K. R. (2003). Electrophysiological correlates of anterior cingulate function in a go/no-go task: effects of response conflict and trial type frequency. Cognitive, Affective, and Behavioral Neuroscience, 3(1), $17-26$.

Pagnini, F., Bercovitz, K., \& Langer, E. (2016). Perceived control and mindfulness: Implication for clinical practice. Journal of Psychotherapy Integration, 26, 91-102.

Pelli, D. G. (1997). The VideoToolbox software for visual psychophysics: Transforming numbers into movies. Spatial Vision, 10, 437-442.

Righi, S., Mecacci, L., \& Viggiano, M. P. (2009). Anxiety, cognitive selfevaluation and performance: ERP correlates. Journal of Anxiety Disorders, 23(8), 1132-1138.

Rubia, K., Russell, T., Overmeyer, S., Brammer, M. J., Bullmore, E. T., Sharma, T., \& Taylor, E. (2001). Mapping motor inhibition: conjunctive brain activations across different versions of go/no-go and stop tasks. Neuroimage, 13(2), 250-261.

Spiewak, S. (2006). Rozgrzewanie uwagi: Wplyw przeciazenia poznawczego na dostepnosc zasobow: Efekt rozgrzania poznawczego. Przeglad Psychologiczny, 49(1), 291-306.

Spiewak, S. (2013). Rozgrzewanie uwagi - wyczerpywanie woliuleglosc: mechanizmy adaptacji umystu do wysitku poznawczego. Warszawa: Scholar.

Tabachnick, B. G., \& Fidell, L. S. (2007). Using multivariate statistics (5th ed.). Boston: Allyn and Bacon.

Wickens, C. D. (2002). Multiple resources and performance prediction. Theoretical Issues in Ergonomic Science, 3(2), 159-177.

Wickens, C. D. (2007). How many resources and how to identify them? Commentary on Boles et al. and Vidulich and Tsang. Human Factors, 49(1), 53-56. 